\title{
Uniform Closure of Certain Classes of Operators
}

\author{
MAHMOUD M. KUTKUT \\ Dept. of Mathematics, Faculty of Science,
} King Abdulaziz University, Jeddah, Saudi Arabia.

\begin{abstract}
In this article, the author proved that certain classes of operators, namely, classes of spectraloid, convexoid, normaloid, those operators having their spectrum spectral sets and those operators having their numerical ranges spectral set, are uniformly closed and arcwise connected. Moreover, some set theoretical relationships between these classes are also proved.
\end{abstract}

\section{A.M.S. Classification (1980)] : Primary : 47 A 25 \\ Secondary: 47 A 12}

Key words: Hilbert space; operator, convexoid, normaloid, spectraloid, spectral set, numerical range.

\section{Introduction}

We consider an infinite dimensional complex Hilbert space $H$. We denote by $L(H)$, the algebra of all bounded linear operators on $\mathrm{H}$.

For an operator $A \in L(H), \sigma(A)$ denotes the spectrum of $A$. The spectral radius, $r(A)$, is defined by the equality

$$
r(A)=\sup \{|\lambda|: \lambda \in \sigma(A)\} .
$$

If $W(A)$ denotes the numerical range of $A$, then $W(A)=\{\lambda \in \mathbb{C}: \lambda=(A \mathbf{e}, \mathbf{e})$ for some $\|\mathbf{e}\|=1\}$, and the numerical radius $w(A)$ is defined by, $w(A)=\sup \{|\lambda|: \lambda \epsilon$ $\overline{W(A)}\}$.

There are many classes of operators that attract the interest of many mathematicians, like unitary operators, normal operators, compact operators.

The class of normal operators denoted by $N$, has very nice properties, it is uniformly closed, arcwise connected, and moreover, if $A \in N \subset L(H)$, then $A$ satisfies the following 
1. $w(A)=\|A\|$

2. $\overline{W(A)}=\operatorname{ch} \sigma(A)$, where ch means the "Convex hull"

3. $r(A)=w(A)$

None of the above statements characterise the normal operator, since there are non-normal operators satisfy one or more of these properties.

Halmos ${ }^{[1]}$ studied some operators which are not normal and satisfy some of the above properties. Halmos, in his book ${ }^{[1]}$, gave names for those operators in order to classify them.

\section{Definition 1}

If $A \in L(H)$ such that $w(A)=\|A\|$, then $A$ is called normaloid. Let $M=$ set of all normaloid operators on $H \subset L(H)$.

\section{Definition 2}

If $A \in L(H)$ such that $\overline{W(A)}=\operatorname{ch} \sigma(A)$, then $A$ is said to be convexoid. Let $C=\{A \in L(H): \overline{W(A)}=\operatorname{ch} \sigma(A)\}$.

\section{Definition 3}

If $A \in L(H)$ such that $r(A)=w(A)$, then $A$ is spectraloid. Let $S=\{A \in L(H): r(A)$ $=w(A)\}$.

It is known that a normaloid operator is spectraloid; also, a convexoid operator is spectraloid. It is easy to give a normaloid operator which is not convexoid and a convexoid operator which is not normaloid; for these information, see: Halmos ${ }^{11}$ Problem 174.

It is clear that $M \subset S$ and $C \subset S$, moreover $M \cap C \neq \phi$, in fact $N \subset M \cap C$.

\section{Definition 4}

A subset $X$ of the complex plane $\mathbb{C}$ is said to be a spectral set of an operator $A \epsilon$ $L(H)$, if $\sigma(A) \subset X$ and $\|f(A)\| \leq \sup \{|f(z)|: z \in X\}=\|f\|_{X}$, for any rational function $f$ with poles off $X$.

One more nice property of normal operator concerning the concept of spectral set, namely, if $A$ is normal operator then $\sigma(A)$ is a spectral set of $A$, see Berberian ${ }^{[2]}$. Let $T=\{A \in L(H): \sigma(A)$ is a spectral set of $A\}$, and let $R=\{A \in L(H)$ : $\overline{W(A)}$ is a spectral set of $A\}$, it is clear that $N \subset T$.

In this article, the author investigates more properties of those classes of operators. He shall prove that: $N \subset T \subset R \subset M \subset S$, and $T \subset R \cap C \subset M \cap C$, so that he can represent the relations between these classes by the following diagram.

Moreover, he shall prove that all of these classes are uniformly closed and arcwise connected. 


\section{Theorem I}

The class $T$ of all operators on $H$ whose spectrum is a spectral set is uniformly closed and arcwise connected.

\section{Proof}

It is known that an operator $A$ has $\sigma(A)$ as a spectral set if, and only if, $f(A)$ is normaloid operator for every rational function $f$ with poles off $\sigma(A)$, (see: Berberian ${ }^{|2|}$ ). With this in mind, let $\left(A_{n}\right) \subset T$ such that $A_{n}$ converges uniformly to $A$, need to show that $A \in T$. This implies that $f\left(A_{n}\right)$ is normaloid, $\forall n$, i.e., $w\left(f\left(A_{n}\right)\right)=\left\|f\left(A_{n}\right)\right\|$, for every rational function with poles off $\sigma\left(f\left(A_{n}\right)\right)$. Since $A_{n}$ converges uniformly (in norm) to $A, f\left(A_{n}\right)$ converges to $f(A)$, for every analytic function $f$, (by functional calculus); and by continuity of the numerical range (see: Halmos ${ }^{1]}$ Problem 220) $w\left(f\left(A_{n}\right)\right)$ converges to $w(f(A))$.

One can also see that $\left\|f\left(A_{n}\right)\right\|$ converges to $\|f(A)\|$ so that by uniqueness of the limit one obtains that $w(f(A))=\|f(A)\|$ for every rational function with poles off $\sigma(f(A))$, i.e., $\sigma(A)$ is spectral set of $A$, or $A \in T$, which proves the first part of the theorem.

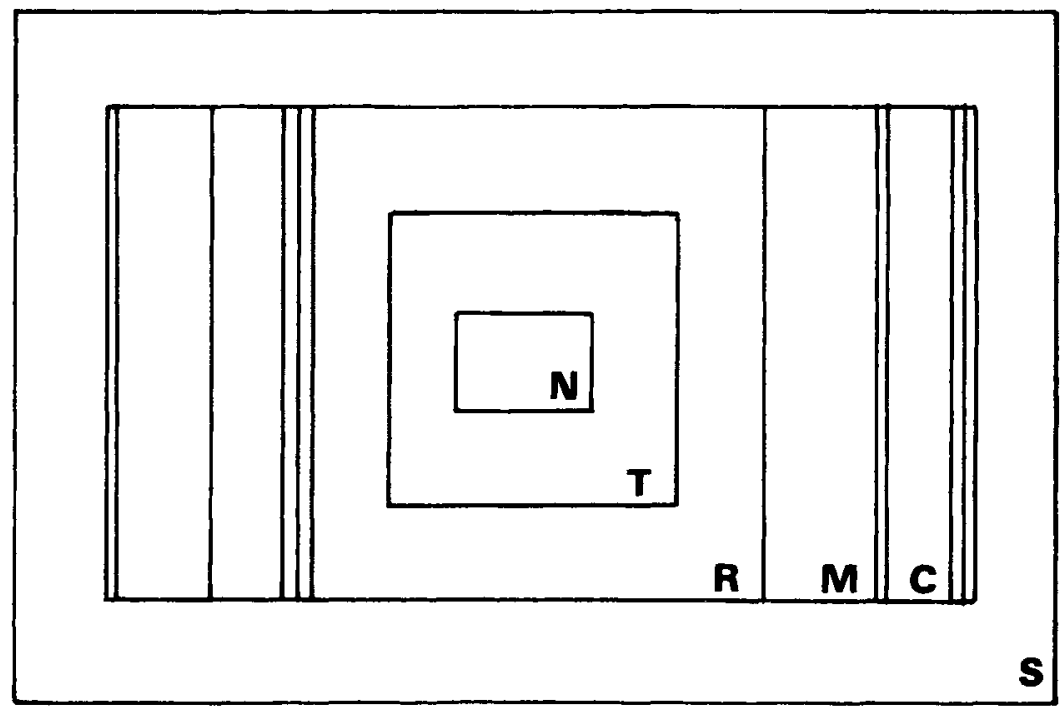

Concerning the other part, let $A \epsilon T$, author is going to prove that $\lambda \cdot A \in \mathrm{T}, \forall \lambda \epsilon$ $\mathbb{C}$. It is known that if $X$ is a spectral set for an operator $A \in L(H), f$ is an analytic function on $X$, then $f(X)$ is a spectral set of $f(A)$, (see: Berberian $\left.{ }^{[2]}\right)$, with this in mind, let $f(x)=\lambda x$, then since $\sigma(A)$ is a spectral set of $A$, then $f(\sigma(A))$ is a spectral set of $f(A)$, i.e., $\lambda \sigma(A)$ is a spectral set of $\lambda A$, and by functional calculus $\lambda \sigma(A)=\sigma(\lambda A)$, thus $T$ is arcwise connected.

\section{Theorem 2}

The class $R$ of all operators on $H$, whose the closure of its numerical range is a spectral set, is uniformly closed and arcwise connected. 


\section{Proof}

Let $\left(A_{n}\right) \subset R$ such that $\left(A_{n}\right)$ converges uniformly to $A \in L(H)$, then by continuity of the numerical range (see: Halmos ${ }^{[1]}$ Problem 220 ) one obtains that $\overline{W\left(A_{n}\right)}$ converges to $\overline{W(A)}$. In order to show that $\overline{W(A)}$ is a spectral set of $A$, it is enough to show that for any polynomial $P,\|P(A)\| \leq\|P\|_{W(A)}$, since $\sigma(A) \subset \overline{W(A)}$. Since $A_{n}$ converges uniformly to $A, P\left(A_{n}\right)$ converges uniformly to $P(A)$, and thus,

$$
\|P(A)\| \leq\left\|P(A)-P\left(A_{n}\right)\right\|+\left\|P\left(A_{n}\right)\right\|,
$$

for $n>N$.

This implies that $\|P(A)\| \leq\left\|P\left(A_{n}\right)\right\|+\epsilon$, on the other hand, $\overline{W\left(A_{n}\right)}$ is a spectral set of $A_{n}$, for every $n$, and therefore,

$$
\left\|P\left(A_{n}\right)\right\| \leq\|P\|_{\overline{W\left(A_{n}\right)}}=\sup \left\{|P(z)|: z \in \overline{W\left(A_{n}\right)}\right\} .
$$

Let $z_{n}=\sup \left\{P(z): z \in \overline{W\left(A_{n}\right)} \quad\right\}$, then $\left(z_{n}\right)$ converges to $z_{0} \in \overline{W(A)}$, where $\left|z_{0}\right| \leq \sup \{|P(z)|: z \in \overline{W(A)}\}$. Combining these inequalities, one should obtain,

$$
\|P(A)\| \leq \epsilon+\|P\|_{\left.\overline{W(A}_{n}\right)} \leq 2 \epsilon+\|P\|_{\overline{W(A)}}
$$

and since $\epsilon$ is arbitrary, we have $\overline{W(A)}$ is a spectral set of $A$ or that $A \epsilon R$.

$R$ is arcwise connected since one has $\overline{W(\lambda A)}=\lambda \overline{W(A)}$ and the result in Berberian ${ }^{[2]}$, namely, $X$ is a spectral set of $A$ then $f(X)$ is a spectral set of $f(A)$, where $f$ is any analytic function on $X$, the function $f(x)=\lambda x$ suffices our need.

\section{Theorem 3}

The class $M$ of all normaloid operators on $H$ is uniformly closed and arcwise connected.

\section{Proof}

If $\left(A_{n}\right) \subset M$, converges uniformly to $A$ then we have $\left\|A_{n}\right\|$ converges to $\|A\|$ and $w\left(A_{n}\right)$ converges to $w(A)$, and since, $w\left(A_{n}\right)=\left\|A_{n}\right\|, \forall n$, we conclude that $w(A)=$ $\|A\|$, i.e., $A \in M$.

For the other part, it is clear that $w(\lambda A)=|\lambda| w(A)$, since $\overline{W(\lambda A)}=\lambda \overline{W(A)}$, moreover, $\|\lambda A\|=|\lambda|\|A\|$ which implies that $\lambda \cdot A$ is normaloid if $A$ is so.

\section{Theorem 4}

The class $C$ of all convexoid operators on $H$, is uniformly closed and arcwise connected. 


\section{Proof}

Let $\left(A_{n}\right) \subset C$, such that $A_{n}$ converges uniformly to $A$, then $\overline{W\left(A_{n}\right)}=c h \sigma$ $\left(A_{n}\right)$, for every $n$; we need to show that $\overline{W(A)}=\operatorname{ch} \sigma(A)$, where $\operatorname{ch} \sigma(A)=$ convex hull of $\sigma(A)$. By the continuity of the numerical range, $\overline{W\left(A_{n}\right)}$ converges to $\overline{W(A)}$. Since $\sigma(A) \subset \overline{W(A)}$, by convexity of $\overline{W(A),} \operatorname{ch} \sigma(A) \subset \overline{W(A)}$. It is enough to show that $\overline{W(A)} \subset \operatorname{ch} \sigma(A)$. For let $\lambda^{\prime} \epsilon \overline{W(A)}$, there is $\lambda_{n} \epsilon$ $\overline{W\left(A_{n}\right)}, \forall n, \lambda_{n}$ converges to $\lambda$, and, $\lambda_{n} \in c h \sigma\left(A_{n}\right)$, for every $n$. It is known that the spectrum is an upper semicontinuous (see: Halmos ${ }^{[1]}$ Problem 103), therefore $\| A$ $A_{n} \|\langle\epsilon, n\rangle N$, since $\sigma(A) \subset \operatorname{ch} \sigma(A)$, one concludes that $\sigma\left(A_{n}\right) \subset \operatorname{ch} \sigma(A)$ for $\left.n\right\rangle$ $N$, so that $d\left(\lambda_{n}, \operatorname{ch} \sigma(A)\right),\left\langle\epsilon, n>N\right.$, which implies that $\lambda_{n} \epsilon \operatorname{ch} \sigma(A)$, change $\epsilon$ arbitrarily to conclude that $\lambda_{n} \in \operatorname{ch} \sigma(A)$ for every $n>N$. But $\lambda_{n}$ converges to $\lambda$ and ch $\sigma(A)$ is compact and thus $\lambda \in \operatorname{ch} \sigma(A)$, i.e. $W(A)=\operatorname{ch} \sigma(A)$, or $A \in C$.

The other part is clear, since one has $\overline{W(\lambda A)}=\lambda \overline{W(A)}$ and ch $\sigma(\lambda A)$ $=\operatorname{ch} \lambda \sigma(A)=\lambda \operatorname{ch} \sigma(A)$, which means the equality of $\overline{W(\lambda A)}$, and $c h \sigma(\lambda A)$, holds if the quality of $\overline{W(A)}$ and $\operatorname{ch} \sigma(A)$ does.

\section{Theorem 5}

The class $\mathrm{S}$ of all spectraloid operators on $H$ is uniformly closed and arcwise connected.

\section{Proof}

Let $\left(A_{n}\right) \subset S$ such that $A_{n}$ converges uniformly to $A$. Then $w\left(A_{n}\right)=r\left(A_{n}\right)$ for every $n$, by continuity of the numerical radius $w\left(A_{n}\right)$ converges to $w(A)$.

Since, the spectrum is upper semicontinuous (see: Halmos ${ }^{[1]}$ page 56 ), one concludes that if $X=\sigma(A)+\epsilon$ and $\left\|A_{n}-A\right\|<\epsilon$ then $\sigma\left(A_{n}\right) \subset X=\sigma(A)+\epsilon$, from which we obtain $r\left(A_{n}\right) \leq r(A)$. But we have also that $w(A)<w\left(A_{n}\right)+\epsilon$, for every $n>N$, so that $w(A)<w\left(A_{n}\right)+\epsilon=r\left(A_{n}\right)+\epsilon<r(A)+\epsilon$, since $\epsilon$ is arbitrary, $w(A) \leq r(A)$. It is known that for any operator $r(A) \leq w(A)$, thus $r(A)=w(A)$ or $A \in S$.

It is easy to show that $S$ is arcwise connected.

\section{Proposition 6}

If $A \in L(H)$ such that $\sigma(A)$ is a spectral set of $A$, then $A$, is convexoid, i.e., $T \subset C$.

\section{Proof}

Since $\sigma(A) \subset \overline{W(A)}$ then $c h \sigma(A) \subset \overline{W(A)}$. It is known that any super set of a spectral set of a given operator is also a spectral set for that operator (see: Berberian ${ }^{[2]}$ ). Therefore, since $\sigma(\mathrm{A})$ is a spectral set of $A$ then $\operatorname{ch} \sigma(A), \overline{W(A)}$ are so. But it is known also that, if $\overline{W(A)}$ is a spectral set, it is the intersection of all convex spectral sets of $A$, (see: Williams ${ }^{|3|}$ ), thus $\overline{W(A)} \subset \operatorname{ch} \sigma(A)$, which means that $A$ is convexoid. 


\section{Proposition 7}

If $A \in L(H), \sigma(A)$ is spectral set of $A$ then $\overline{W(A)}$ is a spectral set of $A$, i.e. $T \subset R$.

\section{Proof}

Clear (see: proof of the previous proposition).

\section{Proposition 8}

If $A \in L(H)$ such that $\overline{W(A)}$ is a spectral set of $A$, then $A$ is normaloid, i.e., $R \subset M$.

\section{Proof}

It is known that for any operator $A \in L(H), w(A) \leq\|A\|$, (see: Halmos ${ }^{[1]}$ Problem 103). Since $\overline{W(A)}$ is a spectral set of $A$, we have $\|\gamma(A)\| \leq\|\gamma\|_{\overline{W(A)}}$, for any rational function $\gamma$, with poles outside $\overline{W(A)}$. In particular, for $\gamma(z)=z$, one obtains,

$$
\begin{aligned}
\|A\| \leq\|\gamma\|_{\overline{W(A)}} & =\sup \{|\gamma(z)|: z \in \overline{W(A)}\} \\
\leq & \sup \{|z|: z \in \overline{W(A)}\}=w(A),
\end{aligned}
$$

which means that $w(A)=\|A\|$, i.e, $A$ is normaloid.

Remarks:

(1) There is an operator $A$ such that $\overline{W(A)}$ is a spectral set but $\sigma(A)$ is not a spectral set for $A$. This operator is defined by Hartman ${ }^{[4]}$. He defines $A$ to be a bilateral weighted shift with $a_{n}=1 / 2$, for $n<0, a_{n}=1$, for $n \geq 0$ as a sequence of weights. This operator has the following properties:

$$
\begin{aligned}
& \|A\|=1=r(A), \text { where, } \\
& \sigma(A)=\{\lambda \in \mathbb{C}: 1 / 2 \leq|\lambda| \leq 1\} .
\end{aligned}
$$

The operator $A$ is hyponormal, and $\sigma(A)$ is not a spectral set of $A$.

Since $A$ is a contraction, the closed uit disc $D$ is a spectral set of $A$.

But $\operatorname{ch} \sigma(A)=\operatorname{ch}\{\lambda \mathbb{C}: 1 / 2 \leq|\lambda| \leq 1\}=D$, i.e., $\operatorname{ch} \sigma(A)$ is a spectral set of $A$, thus, $\overline{W(A)}$ is a spectral set of $A$.

We conclude that $A \in R$, but $A \notin T$.

(2) There is a normaloid operator $A$ such that $\sigma(A)$ is not a spectral set of $A$. 


\section{Indeed:}

Let $K=\left[\begin{array}{ll}0 & 0 \\ 1 & 0\end{array}\right]$ and define $A=\left[\begin{array}{ll}\mathbf{K} & 0 \\ 0 & 1\end{array}\right]$, this operator has the following properties:

$\|A\|=1, \overline{W(A)}=\operatorname{ch}(D(0,1 / 2) \mathrm{U}\{1\})$, where $D(0,1 / 2)$ is the closed disc with radius $1 / 2$.

Thus $\sigma(A)=\{0,1\}$ and $\operatorname{ch} \sigma(A)=[0,1]$, therefore $A$ is normaloid.

Define $f$ to be $f(z)=z-z^{2}$ then $\|f\|_{[0,1]}=\frac{1}{4}$, and $\|f(A)\|_{[0,1]}=\|K\|=1$.

This implies that $\operatorname{ch} \sigma(A)$ is not a spectral set of $A$ and thus $\sigma(A)$ is not a spectral set of $A$.

We conclude that $A \in M$ but $A \notin T$.

(3) There is a convexoid operator $A$ such that $\overline{W(A)}$ is not a spectral set of A.

\section{Indeed :}

Let $K=\left[\begin{array}{ll}0 & 0 \\ 1 & 0\end{array}\right]$ and $B$ be a normal operator such that $\sigma(B)=D(0,1 / 2)$, the closed disc with center 0 and radius $1 / 2$.

Define $A=\left[\begin{array}{ll}K & 0 \\ 0 & B\end{array}\right]$, this operator has the following properties:

$$
\sigma(A)=D(0,1 / 2) \text {, and } \overline{W(A)}=\operatorname{ch} D(0,1 / 2) \text {, thus } A \text { is convexoid. Since }\|A\|=1 \text {, }
$$

for $f(z)=z$ on $\overline{W(\mathrm{~A})}$, and thus $\|f(A)\|=\|A\|=1$. But, $\|f\|_{\overline{W(A)}}=\sup _{z \in \overline{W(A)}}|z|=$

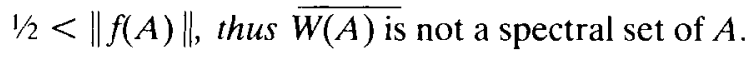

We conclude that $A \in C$, but $A \notin R$.

\section{Acknowledgement}

The author would like to thank the referee for his useful suggestions.

\section{Symbols Appendix}
A : Bounded linear operator on $H$.
$C$ : Class of all convexoid operators on $H$.
$\operatorname{ch}(\sigma(A)) \quad$ : Convex hull of $\sigma(A)$
$\sigma(A) \quad$ : Spectrum of $A$.
e : Vector in $H$.
$f \quad$ : Analytic function on $X$. 


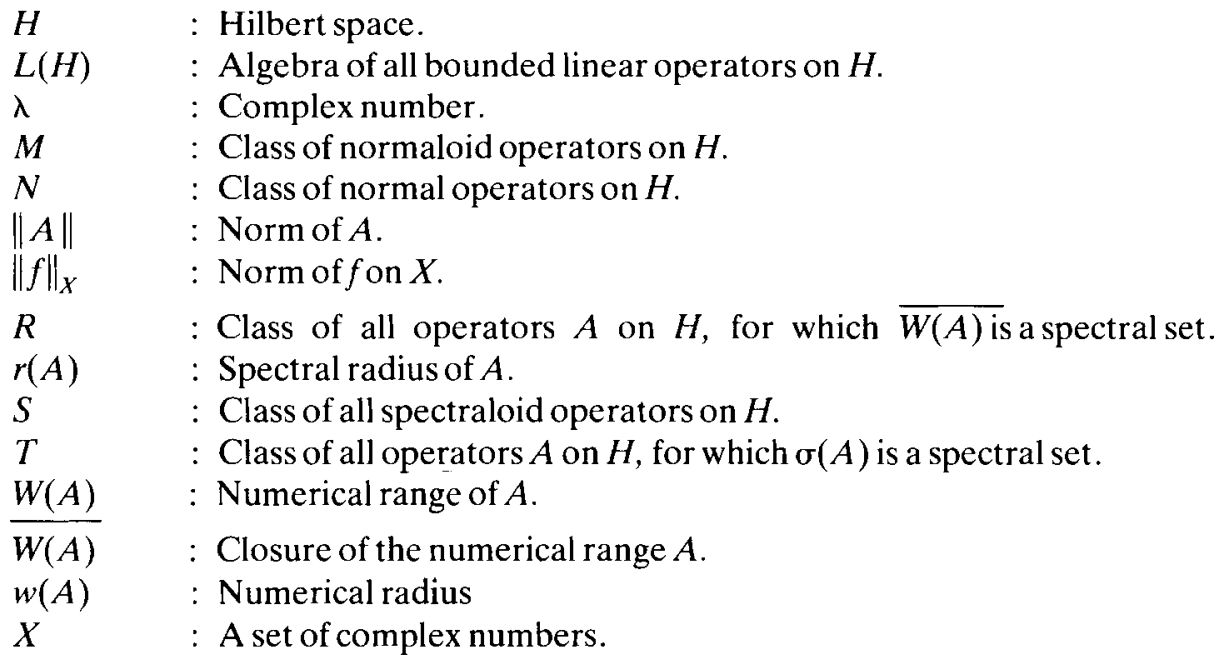

\section{References}

[1] Halmos, P.R., A Hilbert Space Problem Book, van Nostrand, N. Y. (1967).

[2] Berberian, S.K., Lectures in Functional Analysis and Operator Theory, Springer-Verlag, N.Y. (1979).

[3] Williams, J.P., Minimal spectral sets of compact operators, Acta, Sci. Math. 28: 93-106 (1967).

[4] Hartman, Jim, A hyponormal Weighted Shift Whose Spectrum is not a spectral set, J. Operator Theory 8: 401-403 (1982). 


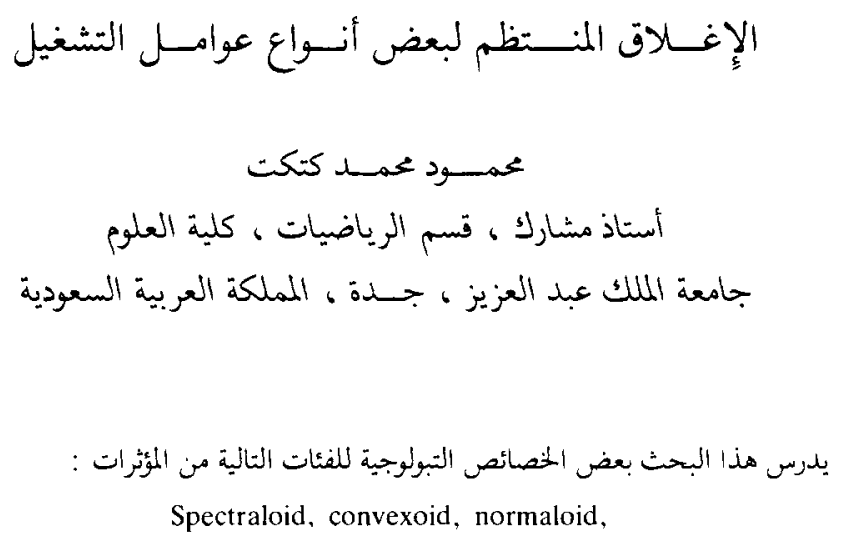

